\title{
Predicting hepatic failure with a new diagnostic technique by preoperative liver scintigraphy and computed tomography: a pilot study in 123 patients undergoing liver resection
}

\author{
Naokazu Chiba ${ }^{1,5^{*}}$, Motohide Shimazu², Kiminori Takano ${ }^{1}$, Go Oshima ${ }^{1}$, Koichi Tomita ${ }^{1}$, Toru Sano ${ }^{1}$, \\ Masaaki Okihara', Yosuke Ozawa', Kosuke Hikita' ${ }^{1}$, Takahiro Gunji', Yuta Abe ${ }^{3}$, Kiyoshi Koizumi ${ }^{4}$ \\ and Shigeyuki Kawachi ${ }^{\top}$
}

\begin{abstract}
Background: A novel index, total liver LU15, has been identified as a surrogate marker for liver function. We evaluated the ability of preoperative remnant liver LU15 values to predict postoperative hepatic failure.

Methods: Preoperative risk factors for postoperative hepatic failure and remnant liver LU15 were evaluated in 123 patients undergoing liver resection for several diseases from September 1st, 2007 to December 1st, 2016. We calculated the remnant liver LU15 value from the total liver LU15 value and the functional remnant liver ratio. Risk factors for postoperative hepatic failure was determined by univariate and multivariate analysis.

Results: Hepatic failure grade B/C developed postoperatively in six patients of seven patients within Makuuchi criteria / without criteria for remnant liver LU15. Operative time $(p=0.0242)$ and criteria for remnant liver LU15 $(p=0.0001)$ were prognostic factors for hepatic failure according to the univariate analysis. And criteria for remnant liver LU15 ( $p=0.0009$ ) was only prognostic factor by multivariate analysis.
\end{abstract}

Conclusion: Based on the findings form this pilot study, it appears that patients with a remnant liver LU15 value of 13 or less may have a high risk of postoperative hepatic failure.

Keywords: Remnant liver LU15, Hepatic failure, ${ }^{99 \mathrm{~m}}$ Tc-labelled galactosyl human serum albumin liver scintigraphy

\section{Background}

Before a hepatectomy, it is necessary to evaluate liver function and estimate the function of the future liver remnant. Assessment of hepatic functional reserve is one of the most important issues in hepatic resection [1-4]. This is especially true for patients with both hepatocellular carcinoma (HCC) and liver cirrhosis, or both hilar cholangiocarcinoma and jaundice, that often need an extended hepatectomy. To avoid a resection that would likely lead

\footnotetext{
* Correspondence: nchiba0632@yahoo.co.jp

'Department of Digestive and Transplantation Surgery, Tokyo Medical

University Hachioji Medical Center, Tokyo, Japan

51163 Tatemachi, Hachioji, Tokyo 193-0998, Japan

Full list of author information is available at the end of the article
}

to postoperative hepatic failure, various methods have been developed for the preoperative assessment of liver function. However, some of these are complex and require calculations involving multiple formulas. To estimate the hepatic functional reserve, ${ }^{99 \mathrm{~m}} \mathrm{Tc}$-labelled diethylene triamine pentaacetate-galactosyl-human serum albumin $\left({ }^{99 \mathrm{~m}} \mathrm{Tc}-\mathrm{GSA}\right)$, a radiopharmaceutical that binds specifically to the hepatic asialoglycoprotein receptor (ASGP-R) has been developed and used clinically to estimate hepatic function $[5,6]$. Because ASGP-R is a natural superficial antigen of viable hepatocytes, the uptake of ${ }^{99 \mathrm{~m}} \mathrm{Tc}-\mathrm{GSA}$ is independent of biochemical processes and allows direct estimation of the functioning hepatocyte mass [7]. In addition, the distribution of ${ }^{99 \mathrm{~m}} \mathrm{Tc}-\mathrm{GSA}$ in the liver is not 
dependent on liver blood flow [8]. Koizumi et al. reported that several parameters for ${ }^{99 \mathrm{~m}} \mathrm{Tc}$-GSA liver scintigraphy were estimated and that LU3 and GSAR15 were interesting and promising parameters for assessing liver function [9].

When a hepatic resection involves more than two sections, evaluation of future functional reserve by using 99mTc-GSA and computed tomography (CT) combined fusion images is currently supposed to be more accurate than a combined estimation using an indocyanine green (ICG) retention test and CT [10]. In this study, remnant liver LU15, which was considered the remnant hepatic functional reserve, was estimated using ${ }^{99 \mathrm{~m}} \mathrm{Tc}-\mathrm{GSA}$ single-photon emission computed tomograpic (SPECT) scintigraphy and $\mathrm{CT}$ fusion images. In addition, the functional reserve of the future liver remnant was also estimated before surgery by using fusion images obtained from contrast-enhanced CT and ${ }^{99 \mathrm{~m}} \mathrm{Tc}-\mathrm{GSA}$ SPECT, and was used to predict liver failure after a hepatic resection. A retrospective study was performed to determine whether the remnant liver LU15 value could enable a final decision regarding a hepatectomy in order to avoid postoperative hepatic failure.

\section{Methods}

\section{Patients}

From September 1st, 2007 to December 1st, 2016 after a

${ }^{99 \mathrm{~m}}$ Tc-labelled galactosyl-human serum albumin liver scintigrapy, a liver resection without biliary tract reconstruction was performed in 123 patients in our department, including 72 patients with HCC, 12 with cholangiocarcinomas, and 35 with metastatic cancers (Table 1). A preoperative portal vein embolization was performed in 21 patients, because their future remnant liver volume was expected to be too small. Liver pathology was classified according to the International Association for the Study of the Liver [11], and the surgical resections were performed according to the liver anatomy of Couinaud [12].

Preoperatively, each patient was evaluated using ${ }^{99 \mathrm{~m}}$ Tc-GSA scintigraphy, conventional liver biochemistry tests, Child-Pugh grading, and a 15 -min retention rate of indocyanine green (ICGR15) test, and the liver volume was measured from $\mathrm{CT}$ scans.

\section{Calculation of remnant liver LU15}

All the patients received $3 \mathrm{mg}$ of ${ }^{99 \mathrm{~m}} \mathrm{Tc}$-GSA (185 MBq; Nihon Medi-Physics, Nishinomiya, Japan) as a bolus injection into the antecubital vein. Total liver LU15 was calculated from the cumulative liver uptake of the tracer 15 to 16 min after an injection of a radiotracer. The hepatic SPECT images were acquired after the dynamic study. Data analyses were performed by creating a ROI of the liver and heart and then by drawing their time- activity curves. Each set of projection data was obtained in a $128 \times 128$ matrix, and 72 projections were acquired. Total LU15 represents the percentage of the integral cumulative count in the liver for 1-min period from 15 to $16 \mathrm{~min}$ after tracer injection to total injected dose. Remnant liver LU15 was calculated as an index or residual liver function by applying the following equation; Remnant liver LU15 $=$ Total LU15 $\mathrm{x}$ residual count ratio. In the recent cases, with the use of the SPECT images by scintigraphy and a three-dimensional (3D) image of the liver constructed by Synapse Vinscent (FujiFilm, Japan), the regional LU15 of the predicted remnant liver (remnant liver LU15) was calculated according to the operative procedures (Fig. 1). Remnant liver LU15 values of 13.0 and more were considered to represent a remnant liver with good liver function, according to Koizumi et al. $[9,13]$.

\section{Postoperative hepatic failure}

Postoperative hepatic failure was defined according to the International Study Group of Liver Surgery (ISGLS) classification [14]. The group also differentiated the severity of postoperative liver failure into three different grades from $\mathrm{A}$ to $\mathrm{C}$, depending on the level of treatment needed. In this study, grade $B$ and $C$ was defined postoperative hepatic failure. Grade B suggests the degree of liver failure needed clinical management but not invasive therapy; grade $\mathrm{C}$ is acute postoperative liver failure requiring invasive treatment [14].

\section{Operative procedure}

The operative procedures included the following; partial resection, segmentectomy, monosectionectomy (eg, right anterior sectionectomy, bisegmentectomy), hemihepatectomy (eg, right or left hepatectomy) and trisectionectomy (eg, right or left trisectionectomy), according to the Brisbane Nomenclature from the International Hepato-PancreatoBiliary Association [15].

\section{Statistical analysis}

The Mann-Whitney U test was used to examine differences in the laboratory test results. The Fisher's exact test or the $\chi^{2}$ test was used to compare categorical data between the groups. Correlations between the remnant liver LU15 criteria and the other laboratory test results were determined using the Pearson's correlation coefficient. Odds ratios were used to estimate the relative risk of postoperative hepatic failure. Logistic regression was used for a univariate analysis, while a multiple logistic regression analysis was used for multivariate analysis. For a multivariate analysis, variables with significant $(p<0.05)$ by the univariate analysis were evaluated. $P$ values of less than 0.05 were considered significant. 
Table 1 Patients characteristics

\begin{tabular}{|c|c|}
\hline & No. of patients $(n=123)$ \\
\hline Gender ratio M:F & $M: F=87: 36$ \\
\hline Median age (range) & $70(23-85)$ \\
\hline \multicolumn{2}{|l|}{ Disease } \\
\hline $\mathrm{HCC}$ & 72 \\
\hline $\mathrm{CCC}$ & 12 \\
\hline Metastatic & 35 \\
\hline Others & 4 \\
\hline Child-Pugh grade $A: B$ & $116: 7$ \\
\hline Portal vein occlusion (+) & 21 \\
\hline \multicolumn{2}{|l|}{ Operation procedure } \\
\hline Partial resection & 15 \\
\hline Segmentectomy & 18 \\
\hline Mono-sectionectomy & 31 \\
\hline Hemihepatectomy & 53 \\
\hline Tri-sectionectomy & 6 \\
\hline $\mathrm{ICG}_{15} \mathrm{R}$ & $12.1(0.3-30.7)$ \\
\hline \multicolumn{2}{|l|}{ Makuuchi criteria } \\
\hline Within & 96 \\
\hline Without & 27 \\
\hline \multicolumn{2}{|c|}{ Parameter from liver scintigraphy } \\
\hline LHL15 & $0.918(0.703-0.977)$ \\
\hline $\mathrm{HH} 15$ & $0.623(0.419-0.906)$ \\
\hline LU15 & $26.7(10.3-43.5)$ \\
\hline remnant liver LU15 & $17.0(3.7-43.2)$ \\
\hline \multicolumn{2}{|c|}{ Criteria for remnant liver LU15 } \\
\hline Within & 110 \\
\hline Without & 13 \\
\hline \multicolumn{2}{|c|}{ Postoperative hepatic failure } \\
\hline grade $B$ & 5 \\
\hline grade $\mathrm{C}$ & 2 \\
\hline
\end{tabular}

HCC Hepatocellular carcinoma, CCC Cholangiocellular carcinoma

\section{Results}

The mean age of the patients was 70 years, with a range of 23 to 85 years. There were 87 (71\%) male and 36 (29\%) female patients, yielding a male:female ratio of 2:1; the patients' Child-Pugh grades included A (94\%) and B (6\%). The surgical procedures consisted of a partial resections (15), segmentectomy (18), monosectionectomy (31), hemihepatectomy (53), and trisectionectomy (6) (Table 1). In 21 patients with portal vein occlusion, remnant liver volume increased from $490 \mathrm{~g}$ to $607 \mathrm{~g}$.

Ninety six patients within Makuuchi criteria and 27 patients developed without Makuuchi criteria (Fig. 1). 13 patients of all 96 patients within Makuuchi criteria developed without criteria for remnant liver LU15. And
27 patients without Makuuchi criteria were within criteria for remnant liver LU15. Total seven patients developed potoperative hepatic failure over grade B. Table 2 shows the details of seven patients developed postoperative hepatic failure. Two patients developed hepatic failure grade $\mathrm{C}$ were performed sgmentectomy, S7/8 segmentactomy and S8 segmentectomy. All two patients died of a hepatic failure while still in the hospital.

Table 3 shows the hazard ratios (HR) for the candidate risk factors associated with postoperative hepatic failure as calculated by the univariate and multivariate analysis. Although serum albumin concentration, ICGR15, aspartate aminotransferase (AST), alanine aminotransferase (ALT), and the other factors including the ICGR 15 criteria by Makuuchi, et al. were not risk factors, operative time and the criteria for remnant liver LU15 were risk factors for postoperative hepatic failure by univariate analysis. And the criteria for remnant liver LU15 was an only risk factor for postoperative hepatic failure by multivariate analysis.

\section{Discussion}

In this study, we attempted to develop a preoperative risk marker for predicting postoperative hepatic failure in patients with any diseases that were candidates for a hepatic resection using $99 \mathrm{mTc}-\mathrm{GSA}$ scintigraphy. After a bolus intravenousinjection of a radiotracer, sequential anterior abdominal $128 \times 128$ matrix size images, including the liver and heart, were acquired every $20 \mathrm{~s}$ for 20 min. The parameters; HH15, LHL15 and LU15 were calculated from the time-activity curves. Two parameters, HH15 and LHL15, should be used together as complementary indications of liver function, because these parameters seem to reach a plateau value in cases of severe liver dysfunction in $\mathrm{HH} 15$ and in cases of liver function improvement in LHL15. In this study, HH15 and LHL15 had week correlation with ICGR15 (data not shown), and the criteria for LHL15 and HH15 were not risk factors for postoperative hepatic failure in univariate analysis. Therefore, HH15 and LHL15 might not be suitable surrogate marker for postoperative hepatic failure. Because LU15 has the advantages of a wider range and a higher value level, as well as better correlation with that of other parameters except ICGR15, LU15 is expected to be the best parameter for regional liver function and seem to be the good surrogate marker for postoperative hepatic failure.

Measurement of the remnant liver volume using planar images obtained by ${ }^{99 \mathrm{~m}} \mathrm{Tc}-\mathrm{GSA}$ scintigraphy is not accurate, but by creating a cut line in each section of the transaxial or frontal SPECT image, precise measurement of the remnant liver hepatic binding concentration is possible [16]. The amount of remnant liver LU15 could be calculated more correctly before surgery by 


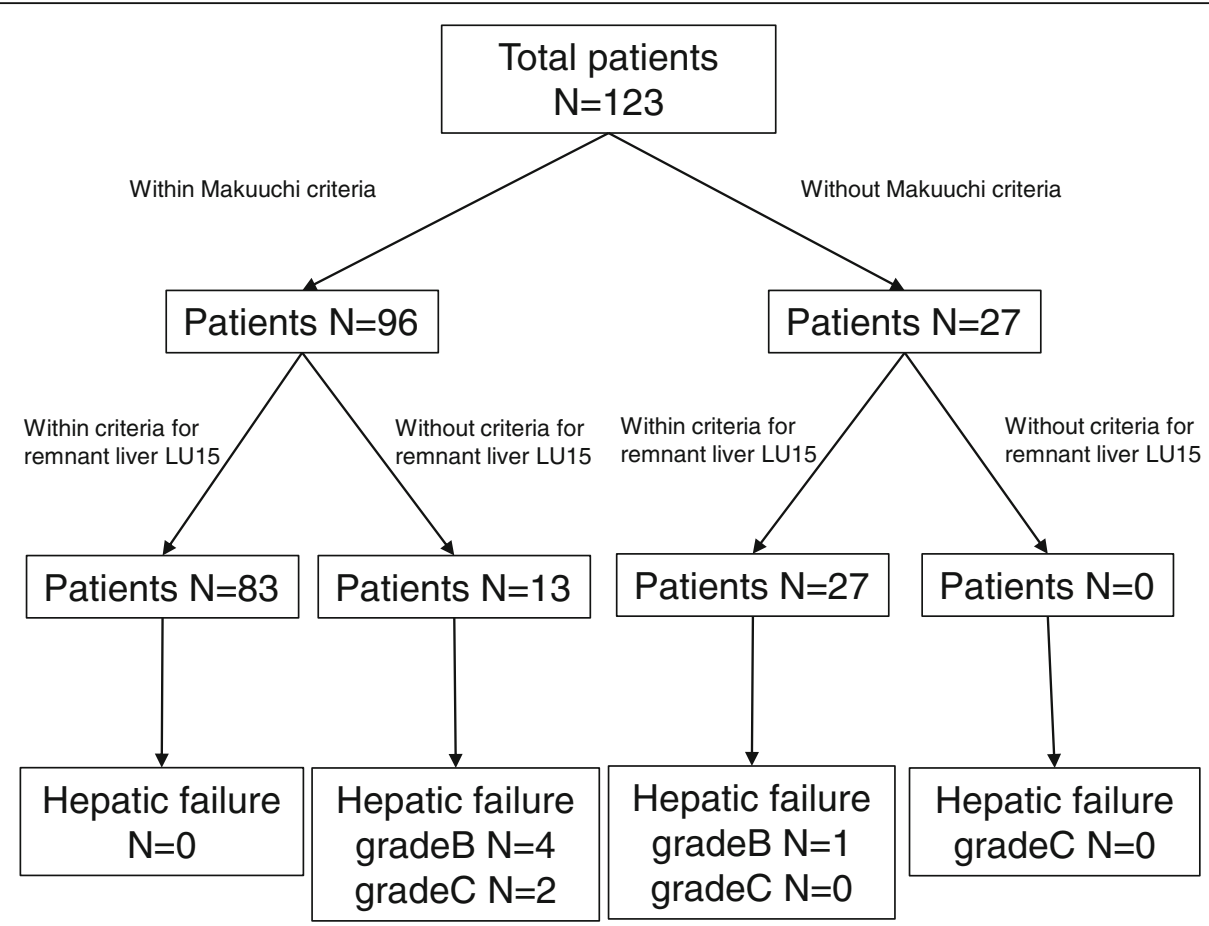

Fig. 1 Distribution of patients according to Makuuchi criteria and remnant liver LU15 criteria. All 123 patients were distributed into 96 patients within Makuuchi crietaria and 27 patients without Makuutchi criteria. And 13 patients of 96 patients developed without remnant liver LU15 criteria and all 27 patients without Makuuchi criteria were developed within remnant liver LU15 criteria

using fusion images of ${ }^{99 \mathrm{~m}}$ Tc-GSA SPECT and contrastenhanced CT scans with the Vinsent software. By using these fusion images, any resection area (partial resection, sub-segmentectomy, and any sectionectomy) could be drawn manually on SPECT scans on the basis of the anatomy observed on $\mathrm{CT}$, permitting calculation of the volume of the future liver remnant. Moreover, remnant liver LU15 could be evaluated in cases with obstructive jaundice (for example, hilar cholangiocarcinoma) or a post portal vein embolization, because the hepatic asialoglycoprotein receptor is not affected by jaundice or a portal vein embolization. Thus, radioactivity within the volume of the future liver remnant could be estimated and the remnant liver LU15 values could be calculated.
Many authors have reported methods for assessing liver volume and function using a standard CT, ${ }^{99 \mathrm{~m}}$ Tc-GSA SPECT, or both $[17,18]$. In HCC with liver cirrhosis, chronic hepatitis or fibrosis, hepatic dysfunction in the hepatic segment or lobe containing HCC was greater than that of the segments or lobe without HCC because the liver parenchyma around the tumor was damaged by mechanical compression, possibly the result of tumor compression of the vessels and bile ducts. If hepatic CT and SPECT images obtained using ${ }^{99 \mathrm{~m}} \mathrm{Tc}-\mathrm{GSA}$ were compared, the defect seen on the SPECT images was larger than that of the tumor seen on the CT image. On the other hand, for cases of hilar cholangiocarcinoma with high serum bilirubin levels, we clarified the usefulness of

Table 2 Patients with postoperative hepatic failure grade B/C

\begin{tabular}{|c|c|c|c|c|c|c|c|c|c|}
\hline Age & Gender & Disease & Child-Pugh & PTPE & $\mathrm{ICG}_{15} \mathrm{R}$ & LU15 & Remnant liver LU15 & Operative procedure & Postoperative complication \\
\hline 68 & $\mathrm{~F}$ & $\mathrm{CCC}$ & A & $(-)$ & 9.5 & 16.7 & 11.5 & Left Hemi. & HF grade $B$ \\
\hline 73 & $\mathrm{~F}$ & $\mathrm{HCC}$ & A & $(-)$ & 12.3 & 15.2 & 12.6 & Left Hemi. & HF grade B \\
\hline 68 & M & $\mathrm{HCC}$ & A & $(-)$ & 17.8 & 14.1 & 12.1 & Left Hemi. & HF grade B \\
\hline 71 & M & $\mathrm{HCC}$ & A & $(-)$ & 15.0 & 12.6 & 10.7 & S8 Seg. & HF grade $B$ \\
\hline 72 & M & $\mathrm{HCC}$ & A & $(-)$ & 7.2 & 26.3 & 12.8 & S7/8 Seg. & HF grade $\mathrm{C}$ \\
\hline 85 & M & $\mathrm{HCC}$ & A & $(-)$ & 28.5 & 16.1 & 12.3 & S8 Seg. & HF grade $\mathrm{C}$ \\
\hline 73 & M & $\mathrm{HCC}$ & A & $(+)$ & 11.0 & 31.1 & 18.8 & Right Tri. & HF grade $B$ \\
\hline
\end{tabular}

CCC Cholangio carcinoma, HCC Hepatocellular carcinoma, Hemi. Hemihepatectomy, Seg. Segmentectomy, Tri. Trisectionectomy, HF Hepatic failure 
Table 3 Prognostic factor for postoperative hepatic failure

\begin{tabular}{|c|c|c|c|}
\hline Univariate analysis & Odds ratio & $95 \% \mathrm{Cl}$ & $p$-value \\
\hline Age & 1.175 & $0.950-1.351$ & 0.1239 \\
\hline \multicolumn{4}{|l|}{ Sex } \\
\hline Male & 1 & & \\
\hline Female & 1.024 & $0.189-5.538$ & 0.9779 \\
\hline \multicolumn{4}{|l|}{ Child-Pugh grade } \\
\hline A & 1 & & \\
\hline B & 2.944 & $0.304-6.540$ & 0.3514 \\
\hline ICG R15 & 1.001 & $0.996-1.007$ & 0.5973 \\
\hline Total billirubin & 0.004 & $0.001-7.003$ & 0.1451 \\
\hline Albumin & 0.214 & $0.019-3.656$ & 0.2870 \\
\hline AST & 0.983 & $0.921-1.049$ & 0.6065 \\
\hline Prothoronbin test & 0.946 & $0.847-1.057$ & 0.3270 \\
\hline Intraoperative blood loss & 1.000 & $0.998-1.001$ & 0.6229 \\
\hline Operation time & 1.137 & $1.017-1.271$ & 0.0242 \\
\hline LHL 15 & 0.041 & $0.007-15.070$ & 0.3927 \\
\hline $\mathrm{HH} 15$ & 0.074 & $0.009-72.115$ & 0.6485 \\
\hline LU 15 & 0.965 & $0.884-1.054$ & 0.9650 \\
\hline Remnant liver LU 15 & 0.915 & $0.789-1.062$ & 0.2425 \\
\hline \multicolumn{4}{|l|}{ Makuuchi criteria } \\
\hline Within & 1 & & \\
\hline Without & 1.400 & $0.257-7.640$ & 0.6975 \\
\hline \multicolumn{4}{|c|}{ Criteria for remnant liver LU15 } \\
\hline$>13.0$ & 1 & & \\
\hline$<13.0$ & 81.750 & $8.741-764.566$ & 0.000 \\
\hline
\end{tabular}

\begin{tabular}{llll} 
Multivariate analysis & & & \\
Operation time & 1.073 & $0.938-1.226$ & 0.3041 \\
Criteria for remnant liver LU15 & 67.724 & $6.840-670.561$ & 0.0009 \\
\hline
\end{tabular}

${ }^{99 m}$ Tc-GSA volumetry because evaluation of the ICG test was unreliable, as bilirubin competes with hepatic ICG excretion. Further, a diseased liver without drainage of the obstructive bile duct showed poor functioning. Application of volumetry by ${ }^{99 \mathrm{~m}}$ Tc-GSA scintigraphy might address the limitations of the ICG test, as this test can evaluate separated liver functions in any situation or background liver function [19]. In this study, remnant liver volume estimated by CT volumetry was greater than that of ${ }^{99 m}$ Tc-GSA scintigraphy in cases of hilar cholangiocarcinoma (data not shown).

Other studies have evaluated a variety of quantitative liver function tests to predict the risk of postoperative death and complications. Conventional biochemical liver tests, such as serum bilirubin, prothrombin time, and Child-Pugh classification, have only limited value with respect to estimating the hepatocellular reserve.
Yamanaka et al. reported that an ICG test, in combination with a radiologic estimation of the liver volume, was of value for predicting liver failure after a hepatectomy [20]. Kinetic analysis of hepatic ICG uptake has been a useful method to evaluate hepatic function, and has been reported to be a good preoperative predictor of death and complications in patients undergoing a liver resection for HCC [21]. The occasional discrepancy between the ICG clearance values and histologic liver findings are thought to depend mainly on the hepatic blood supply and intra/extrahepatic shunt. First, ICG retention is expected to increase in patients with jaundice, because ICG is excreted into the biliary system. Second, a rare constitutional dye excretory disorder has been reported. Moreover, as a test agent, it is well-known that ICG has some adverse reactions such as shock, nausea, rashes and itching. The ability to make a final decision about the extent of liver resection with an underlying liver disease requires new methods to estimate the functional hepatic reserve in the predicted remnant liver, rather than the entire liver.

\section{Conclusions}

Preoperative remnant liver LU15 values independently predicted hepatic failure following a liver resection for any disease, including $\mathrm{HCC}$ with liver cirrhosis and hilar cholangiocarcinma with obstructive jaundice. It appears that patients with a remnant liver LU15 value of 13 or less may have a high risk of postoperative hepatic failure.

\section{Acknowledgements}

Not applicable.

Funding

All authors had no funding for this research.

Availability of data and materials

All authors and all reviewer had availability of these data and materials.

Authors' contributions

NC, KK and SK participated in the design of the study. NC drafted the manuscript and performed the statistical analysis. SK conceived of the study, and participated in its design and coordination and helped to draft the manuscript. All authors performed operation procedures, read and approved the final manuscript.

Ethics approval and consent to participate

This research data was approved by Tokyo Medical University Hachioji Medical Center Ethics committee (Approval No. H175).

Consent for publication

This research data had consent of publications from all patents.

Competing interests

The authors declare that they have no competing interests.

\section{Publisher's Note}

Springer Nature remains neutral with regard to jurisdictional claims in published maps and institutional affiliations. 


\section{Author details}

'Department of Digestive and Transplantation Surgery, Tokyo Medical University Hachioji Medical Center, Tokyo, Japan. ${ }^{2}$ Department of Surgery, Tama Kyuryo Hospital, Tokyo, Japan. ${ }^{3}$ Department of Surgery, Keio University school of Medicine, Tokyo, Japan. ${ }^{4}$ Department of Radiology, Tokyo Medical University Hachioji Medical Center, Tokyo, Japan. ${ }^{5} 1163$ Tatemachi, Hachioji, Tokyo 193-0998, Japan

\section{Received: 25 July 2017 Accepted: 20 November 2017}

Published online: 18 December 2017

\section{References}

1. Arii S, Yamada Y, Futagawa S, et al. Results of surgical and nonsurgical treatment for small-sized hepatocellular carcinomas: a retrospective and nationwide survey in Japan. The liver cancer study Group of Japan. Hepatology. 2000;32:1224-9.

2. Nagino M, Kamiya J, Nishio $\mathrm{H}$, et al. Two hundred forty consecutive portal vein embolizations before extended hepatectomy for biliary cancer: surgical outcome and long-term follow-up. Ann Surg. 2006;243:364-72.

3. Mitsumori A, Nagaya I, Kimoto S, et al. Preoperative evaluation of functional reserve following hepatectomy by technetium-99m galactosyl human serum albumin liver scintigraphy and computed tomography. Eur J Nuc Med. 1998;25:1377-82.

4. Hayashi $\mathrm{H}$, Beppu T, Okabe $\mathrm{H}$, et al. Functional assessment versus conventional volumetric assessment in the prediction of operative outcomes after major hepatectomy. Surgery. 2015;157:20-6.

5. Onodera Y, Takahashi K, Togashi T, et al. Clinical assessment of hepatic functional reserve using 99mTc DTPA galactosyl human serum albumin SPECT to prognosticate chronic hepatic diseases-validation of the use of SPECT and a new indicator. Ann Nucl Med. 2003;17(3):181-8.

6. Kudo M, Todo A, Ikekubo K, et al. Quantitative assessment of hepatocellular function through in vivo radioreceptor imaging with technetium $99 \mathrm{~m}$ galactosyl human serum albumin. Hepatology. 1993;17:814-9.

7. Kwon AH, Ha-Kawa SK, Uetsuji S, et al. Use of technetium 99m diethylenetriamine-pentaacetic acid-galactosyl-human serum albumin liver scintigraphy in the evaluation of preoperative and postoperative hepatic functional reserve for hepatectomy. Surgery. 1995;117:429-34.

8. Kokudo N, Vera DR, Koizumi M, et al. Recovery of hepatic asialoglycoprotein receptors after major hepatic resection. J Nucl Med. 1999:40:137-41.

9. Koizumi K, Uchiyama G, Arai T, et al. A new liver functional study using Tc-99m DTPA-galactosyl human serum albumin: evaluation of the validity of several functional parameters. Ann Nucl Med. 1992;6:83-7.

10. Sumiyoshi T, Shima $Y$, Okabayashi $T$, et al. Liver function assessment using 99mTc-GSA single-photon emission computed tomography (SPECT)/CT fusion imaging in hilar bile duct cancer: a retrospective study. Surgery. 2016; 160(1):118-26.

11. Anthony PP, Ishak KG, Nayak NC, et al. The morphology of cirrhosis. Recommendations on definition, nomenclature, and classification by working group sponsored by the World Health Organization. J Clin Pathol. 1978:31:395-414.

12. Couinaud C. Surgical anatomy of the liver. Several new aspects. Chirurgie. 1986;112:337-42.

13. Uetake M, Koizumi K, Yagawa A, et al. Use of Tc-99m DTPA galactosyl human serum albumin to predict postoperative residual liver function. Clin Nucl Med. 1999:24(6):428-34.

14. Rahbari NN, Garden OJ, Padbury R, et al. Posthepatectomy liver failure: a definition and grading by the international study Group of Liver Surgery (ISGLS). Surgery. 2011;145:713-24.

15. Kubota K, Makuuchi M, Kusaka K, et al. Measurement of liver volume and hepatic functional reserve as a guide to decision-making in resectiona surgery for hepatic tumors. Hepatology. 1997:26:1176-81.

16. Kwon AH, Ha-Kawa SK, Uetsuji S, et al. Preoperative determination of the surgical procedure for hepatectomy using technetium-99m-galactosyl human serum albumin (99mTc-GSA) liver scintigraphy. Hepatology. 1997;25:426-9.

17. Beppu $T$, Nitta $H$, Hayashi $H$, et al. Effect of branched-chain amino acid supplementation on functional liver regeneration in patients undergoing portal vein embolization and sequential hepatectomy: a randomized controlled trial. J Gastroenterol. 2015:50(12):1197-205.

18. Yoshida M, Beppu T, Shiraishi S, et al. (99m)Tc-GSA SPECT/CT fused images for assessment of hepatic function and hepatectomy planning. Ann Transl Med. 2015;3(2):17.
19. Nanashima A, Yamaguchi H, Shibasaki S, et al. Relationship between indocyanine green test and technetium-99m galactosyl serum albumin scintigraphy in patients scheduled for hepatectomy: clinical evaluation and patient outcome. Hepatol Res. 2004;28:184-90.

20. Yamanaka N, Okamoto E, Oriyama T. A prediction scoring system to select the surgical treatment of liver cancer. Further refinement based on 10 years of use. Ann Surg. 1994;219:342-6.

21. Lau H, Man K, Fan ST, et al. Evaluation of preoperative hepatic function in patients with hepatocellular carcinoma undergoing hepatectomy. Br J Surg. 1997;84:1255-9.

\section{Submit your next manuscript to BioMed Central and we will help you at every step:}

- We accept pre-submission inquiries

- Our selector tool helps you to find the most relevant journal

- We provide round the clock customer support

- Convenient online submission

- Thorough peer review

- Inclusion in PubMed and all major indexing services

- Maximum visibility for your research

Submit your manuscript at www.biomedcentral.com/submit
Biomed Central 